\title{
Death from petrol inhalation after an armoured vehicle rollover
}

\author{
M L Davies, T K Rowlands
}

Emerg Med J 2003;20:494-495

A case is reported of cardiac arrest in a young man after an armoured vehicle rollover accident. The proposed mechanism of death is myocardial sensitisation to endogenous catecholamines after exposure to petroleum fuel.

W e would like to report a case of cardiac arrest in a young fit man. The arrest occurred after an armoured vehicle accident in which the victim sustained no life threatening injuries. The pathophysiology of the arrest is related to organic solvent myocardial sensitiation and will be further alluded to in the history and discussion.

The incident occurred during Exercise Phantom Bugle, one of the British Army's major armoured vehicle exercises. Exercise Phantom Bugle is an armoured infantry battle group exercise deploying onto a major military training area in the United Kingdom. It is conducted four times a year and involves about 1200 troops from infantry, armour, aviation, and support units from across the United Kingdom. The troops are deployed in a variety of military vehicles, most of which are armoured tracked vehicles including Challenger 2 Main Battle tanks, Warrior Armoured Fighting Vehicles, FS 430 Armoured Personnel Carriers and the focus of this report the Sabre CVR (T) armoured reconnaissance vehicle (fig 1). The aim of the exercise is to test the abilities of military commanders in a simulated battle environment.

\section{CASE HISTORY}

The patient, a 37 year old army major was mounted at the commander's position in the turret of a Sabre CVR (T) armoured reconnaissance vehicle at the beginning of the final day of Exercise Phantom Bugle. The vehicle was in the process of positioning itself in preparation for the start of that day's battle, when it lost control of its lower track on a grassy knoll. This resulted in the vehicle toppling over into a ditch during which the patient sustained an injury to his head.

The remaining two occupants of the vehicle were distressed but otherwise uninjured and extracted themselves from the

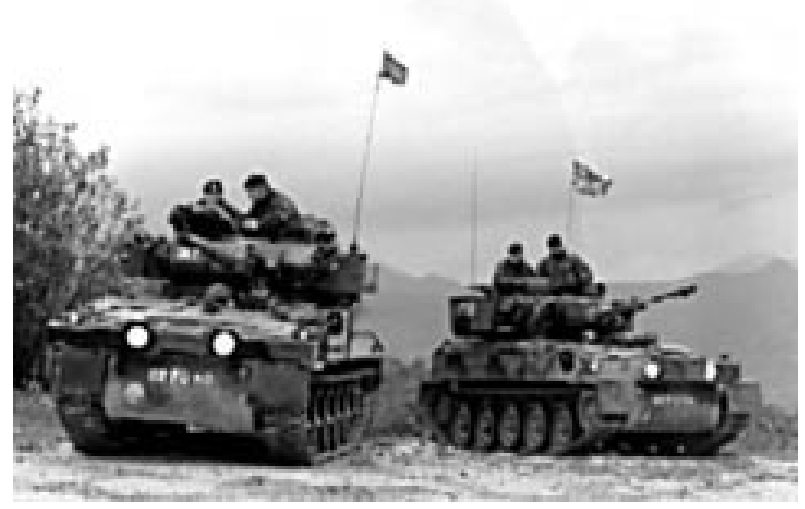

Figure 1 A Sabre CVR (T) armoured reconnaissance vehicle. vehicle. The patient however remained trapped within the turret opening by his leg. He was conscious from time of the accident until he arrested about five minutes later. During this time he was reportedly fully orientated and able to maintain a lucid conversation with the uninjured parties.

During the accident the upturned vehicle had spilt its load of petroleum fuel into the ditch in which the vehicle came to lie. This had the effect of allowing petroleum vapours to accumulate in the air being breathed by the casualty.

The patient told his colleagues that he was "losing it", and fell unconscious, on examination by his fellow soldiers no pulse was palpable, and the patient was extricated urgently from the vehicle and cardiopulmonary resuscitation (CPR) was started according to basic life support protocols and help sought. Two military medical officers, who were positioned with the exercise's command elements, were covering the exercise. They were immediately summoned by radio and arrived at the scene about 15 minutes later to find that two person CPR was in progress.

On arrival the airway was maintained with jaw thrust and oral airway adjunct. Bag-valve-mask ventilation was started together with supplementary oxygen $(15 \mathrm{l} / \mathrm{min})$. CPR was continued, intravenous access obtained and fluid resuscitation begun for presumed traumatic blood loss, resuscitation drugs were administered as per ALS protocol. On examination the casualty was found to have a friction burn injury to his forehead with no other apparent external injuries. Arrangements were made for a Lynx helicopter to evacuate the casualty to the nearest emergency hospital. In the meantime CPR was continued, and the patient packaged with endotracheal intubation and cervical spine triple immobilisation, no defibrillator was available at scene. Transport to hospital took 20 minutes flying time and CPR was continued throughout.

On arrival at the receiving hospital the patient was found to by in ventricular fibrillation and was cardioverted to sinus rhythm on his second shock as per ALS resuscitation algorithm. His Glasgow Coma Score remained 3 despite adequate cardiac output; on further examination blood was found to be coming from the left external auditory meatus. A diagnostic peritoneal lavage was performed, which was negative. Computed tomography of his head on admission revealed global cerebral oedema with signs of raised intracranial pressure, later he was assessed for brain stem death and his ventilatory support was removed the next day.

A postmortem examination was conducted shortly after death and the following findings noted.

\section{Central nervous system}

The skull contained a $3 \mathrm{~cm}$ linear base of skull fracture running transversely in the middle cranial fossa on the left. The brain was generally swollen, no haemorrhages or contusions were seen, and appearances were consistent with diffuse cerebral oedema.

\section{Respiratory system}

The bronchi contained heavily bloodstained fluid, there were bilateral pleural effusions consisting of $500 \mathrm{ml}$ of strawcoloured fluid. The lung parenchyma was markedly congested and oedematous. 


\section{Cardiovascular system}

The ventricles, atria, valves, and endocardium appeared normal, the coronary arteries and aorta were free from atherosclerosis.

\section{Gastrointestinal system}

The stomach contained $50 \mathrm{ml}$ of slightly blood stained fluid, but otherwise normal, the liver had a mottled appearance with areas of pallor on cut section consistent with fatty change and areas of congestion. The remainder of the gastrointestinal system was normal.

The urogenital, lymphoreticular, endocrine and musculoskeletal systems were normal.

Histological examination revealed normal myocardium; congested kidneys, with granular debris in Bowman's space consistent with shock; congested lungs with haemorrhage and oedema; brain tissue revealed no evidence of established ischaemia or axonal retraction balls. Routine toxicological analysis revealed no evidence of any abnormalities.

The cause of death was recorded as:

la Cerebral oedema

lb Cardiac arrhythmia

lc Inhalation of petrol (gasoline) fumes.

\section{DISCUSSION}

Sudden death from inhalation of petroleum distillates is well recognised in misusers of volatile substances. ${ }^{1-3}$ Fatal accidental exposure is usually associated with ingestion of the fuel during syphoning ${ }^{3}$ and fatal exposure during appropriate use is rare. ${ }^{1}$

There are several recognised mechanisms by which death from petroleum exposure may occur. Arrhythmias secondary to sensitisation of myocardium to catecholamines, vasovagal events, CNS respiratory depression, hypoxia, and hypercapnia all have been suggested and some are dependent on the mechanism of administration..$^{1-3}$

The use of defibrillation in this patient's case was delayed because of his remote location and lack of appropriate equipment, earlier defibrillation may have improved outcome, however the use of defibrillation in this environment brings the danger of explosion and probably would not have been feasible from a safety point of view.
The proposed mechanism of death in this case is myocardial sensitisation to endogenous catecholamines and this is supported by the absence of any other significantly life threatening injury and the absence of any evidence to support the other mechanisms that have been proposed above. Vasovagal events are associated with the application of cold liquid or spray to the oropharynx, ${ }^{1}$ CNS respiratory depression would be evident from the history with a gradually reducing level of consciousness and there is no reason that the patient should have become hypoxic or hypercapnic from the history. The authors are unaware of any specific toxicological or biochemical markers, which would support the conclusion that acute inhalation of petrol fumes caused the death.

Death from inhalation of petroleum fumes is rare and death after accidental exposure is even less common. We have reported what we believe is the first case of accidental exposure after a vehicle accident. A programme of dieselisation of military vehicles is currently underway and we hope that because of the lower volatility of diesel that the chances of a recurrence of this case are low within this environment.

\section{Authors' affiliations \\ M L Davies, Department of Surgery, Frimley Park Hospital, Camberley, UK \\ T K Rowlands, Department of Orthopaedics, Peterborough District Hospital, UK \\ Funding: none. \\ Conflicts of interest: none declared.}

Correspondence to: Captain M L Davies, Ministry of Defence Hospital Unit (Frimley Park), Frimley Park Hospital, Portsmouth Road, Camberley, Surrey GU16 5UJ, UK; leighdavies@doctors.org.uk

\section{REFERENCES}

1 Steffe CH, Davis GJD, Nichol KK. A whiff of death: fatal volatile solvent inhalation abuse. South Med J 1996;89:879-84.

2 Chalmers EM. Volatile substance abuse. Med J Aust 1991;154:26974

3 Yorukoglu D, Sen S, Saygin B. Sudden death from inhalation of petrol vapour. Anaesthesia 1999;54:302.

\section{Tension pneumothorax: eyes may be more diagnostic than ears}

\section{S Leigh-Smith, G Davies}

A case is presented of unilateral tension pneumothorax in an awake patient who was seen in prehospital care after a significant fall. Because of extrication difficulties it was 40 minutes after the accident when he was first seen and by this stage the tension pneumothorax was well developed. Many features that are taught as "classic" of tension pneumothorax were absent but various other clinical signs were present to aid the diagnosis, and these are reviewed.
A 14 year old boy fell three storeys into a domestic lift shaft. A prehospital care team of emergency physician and paramedic was immediately dispatched, arriving on scene within seven minutes of the accident. There was no access to the patient as the lower lift doors were shut but he could just be seen from the third floor doors. He was standing up, moving around, and talking although confused and agitated. It was 40 minutes after the accident when full patient access was gained at the lower end of the lift shaft. By this stage he was no longer talking and obviously in severe respiratory distress. He was otherwise alert and confused but 
could just about cooperate with extrication commands. There was no room for a stretcher in the lift shaft. He underwent a rapid extrication in an ambulance chair with a Stifneck collar followed by administration of oxygen.

Examination revealed; a minor occipital contusion but no other external sign of injury, marked agitation, GCS 10 (V1M6E4), moving all four limbs, marked cyanosis, respiratory rate of $30+$, warm and well perfused peripheries, heart rate $135, \mathrm{Spo}_{2}$ unrecordable on 15 litres oxygen with reservoir bag, blood pressure 137/67, central trachea, equal thoracic resonance bilaterally and equal air entry bilaterally but with scattered crepitations.

There was no evidence of flail chest and a provisional diagnosis of unilateral or bilateral tension pneumothorax was made.

Further careful visual inspection in an attempt to lateralise his thoracic injury revealed subtle signs of left sided thoracic hyperexpansion and hypomobility.

Left sided needle thoracocentesis in the 2nd ICS MCL produced a brief hiss of air but with no clinical improvement. Left sided tube thoracostomy was immediately performed after sedation and analgesia with $3 \mathrm{mg}$ midazolam and $20 \mathrm{mg}$ ketamine. This resulted in a large hiss of air that continued for some seconds along with a small haemothorax. $\mathrm{Spo}_{2}$ improved to $90 \%$ with thoracostomy and then $95 \%$ with tube drainage. He underwent uncomplicated rapid sequence intubation then right sided thoracostomy ${ }^{1}$ that revealed no tension on that side. Investigation in hospital revealed a ruptured spleen (removed), left pulmonary contusion, stable lumbar fracture, and left pelvic ramus fracture. He had persistent left sided intrapleural air leak, which subsequently settled on conservative management. After two weeks he was discharged home with normal gross cognitive function.

\section{DISCUSSION}

Tension pneumothorax is one of six conditions listed as "immediately life threatening thoracic injuries" in ATLS ${ }^{2}$ teaching. Rapid treatment is required and as such the diagnosis usually relies on clinical signs. Five standard texts list a variety of signs that are variably present to make the diagnosis. ${ }^{2-6}$ Different emphasis is given to some of these signs in the texts with just two signs (respiratory distress, tachycardia) accepted as being universally present in this disease.

The presence of cyanosis is $\mathrm{FiO}_{2}$ dependent. Gradually developing hypoxia is a consistent feature of developing tension pneumothorax if patients are breathing air. This patient's $\mathrm{Spo}_{2}$ may have been less than $66 \%$ as it has been suggested that this is the highest level at which cyanosis (with a normal haemoglobin) is clinically detectable. ${ }^{7}$

In a patient who is in severe respiratory distress with tension pneumothorax as a provisional diagnosis it is desirable to be able to lateralise the disease clinically. However, the classically taught tracheal deviation is often absent and at best an inconsistent or late finding. ${ }^{3-10}$ Comparing this case report with the signs listed in these standard texts only one ${ }^{3}$ gives over-inflation and splayed ribs as signs of the disease. Although not commonly reported ipsilateral hyperinflation and hypomobility have been mentioned in other case reports. ${ }^{111}$ Hyperinflation (along with ipsilateral diaphragmatic depression) has however been noted ipsilaterally in chest radiographs of tension pneumothorax. ${ }^{12}$ This ipsilateral hyperinflation is a late sign of tension as earlier on there will be hypoexpansion and hypomobility due to collapse of the lung and pain limitation of thoracic movement.

The other commonly taught lateralising signs of hyperresonance and decreased air entry have been previously noted to be absent even in an advanced state of the disease process. ${ }^{913}{ }^{14}$ Other authors have commented on the poor reliability in eliciting chest signs by chest physicians ${ }^{15}$ and doctors in the accident and emergency department ${ }^{16}$-let alone in prehospital care.

This case report also adds further to the existing evidence base that needle thoracocentesis may be an unreliable treatment for tension pneumothorax. ${ }^{17-24}$

In conclusion, tension pneumothorax may be a difficult diagnosis to make with variable presentation of signs. Deliberate and careful visual inspection of the chest wall may reveal useful clinical signs. In a supine patient this must be done by getting down to the level of the patient. Careful looking along the line of the thorax may detect subtle differences in thoracic size and mobility.

\section{Authors' affiliations}

S Leigh-Smith, Defence Medical Services, UK

G Davies, Department of Emergency Medicine and Pre-hospital Care, Royal London Hospital, UK

Correspondence to: Mr S Leigh-Smith, Flat 3F1, 40 Warrender Park Terrace, Edinburgh EH9 1EB, UK; simonlsuk@aol.com

Accepted for publiction 20 January 2003

\section{REFERENCES}

1 Deakin CD, Davies G, Wilson A. Simple thoracostomy avoids chest drain insertion in prehospital trauma. J Trauma 1995;39:373-4.

2 American College of Surgeons. Advanced trauma life support. 6th edn. Chicago: American College of Surgeons, 1997

3 Greaves I, Porter K, Ryan J. Trauma care. London: Arnold, 2001:57.

4 Rosen P, Barkin R. Emergency medicine. 5th edn. St Louis: Mosby, 2002:388

5 Tintinalli JE, Kelen GD, Stapczynski JS. Emergency medicine. 5th edn. New York: McGraw Hill, 2000.

6 Wyatt JP, Illingworth RN, Clancy M, et al. Oxford handbook of accident and emergency medicine. Oxford: Oxford University Press, 1999:347.

7 Bray JJ, Cragg PA, Macknight ADC, et al. Lecture notes on human physiology. 3rd edn. Oxford: Blackwell Science, 1994.

8 Askins DC. Spontaneous tension pneumothorax during sexual intercourse. Ann Emerg Med 1984;13:303-6.

9 Rojas R, Wasserberger J, Balasubramaniam S. Unsuspected tension pneumothorax as a hidden cause of unsuccessful resuscitation. Ann Emerg Med 1983;12:411-12.

10 Holloway VJ, Harris JK. Spontaneous pneumothorax: is it under tension?. J Accid Emerg Med 2000;1 7:222-3.

11 Watts BL, Howell MA. Tension pneumothorax: a difficult diagnosis. Emerg Med J 2001;18:319-20.

12 Teplick SK, Clark RE. Various faces of tension pneumothorax. Postgrad Med 1974;56:87-92.

13 Vermeulen EG, Teng HT, Boxma H. Ventral tension pneumothorax. J Trauma 1997;43:975-6.

14 Werne CS, Sands M. Left tension pneumothorax masquerading as anterior myocardial infarction. Ann Emerg Med 1985; 14:164-6.

15 Spiteri MA, Cook DG, Clark SW. Reliability of eliciting physical signs in examination of the chest. Lancet 1988;i:873-5.

16 Dunlop MG, Beattie TF, Preston PG, et al. Clinical assessment and radiography following blunt chest trauma. Arch Emerg Med 1989;6:125-7.

17 Britten S, Palmer SH, Snow TM. Needle thoracocentesis in tension pneumothorax: insufficient cannula length and potential failure. Injury 1996;27:321-2

18 Britten S, Palmer SH. Chest wall thickness may limit adequate drainage of tension pneumothorax by needle thoracocentesis. J Accid Emerg Med 1996;13:426-7.

19 Conces DJ, Tavver RD, Gray WC, et al. Treatment of pneumothoraces utilising small calibre chest tubes. Chest 1988;94:55.

20 Cullinane DC, Morris JA Jr, Bass JG, et al. Needle thoracostomy may not be indicated in the trauma patient. Injury 2001;32:749-52.

21 Jenkins C, Sudheer PS. Needle thoracocentesis fails to diagnose a large pneumothorax. Anaesthesia 2000;55:925-6.

22 Jones R, Hollingsworth J. Tension pneumothoraces not responding to needle thoracocentesis. Emerg Med J 2002;19:176-7.

23 Mines D, Abbuhl S. Needle thoracostomy fails to detect a fatal tension pneumothorax. Ann Emerg Med 1993;22:1364.

24 Pattison GTR. Needle thoracocentesis in tension pneumothorax insufficient cannula length and potential failure. Injury 1996;27:758. 